\title{
“Esa euforia rusófila": La opinión americana sobre la Unión Soviética durante la Segunda Guerra Mundial y La consagración de la primavera de Alejo Carpentier.
}

\author{
David Becerra Mayor \\ Université catholique de Louvain \\ david.becerra@uclouvain.be
}

\begin{abstract}
The novel La Consagración de la primavera [The Rite of Spring] by Alejo Carpentier describes the favorable opinion of the American people about the Soviet Union during the Second World War as a phenomenon of "Russophile euphoria." This article aims to start from this concept that the novel presents, as a Benjaminian ragpicker, rediscover the historical residues that have remained on the margins of official history. Reviewing the cultural context, the books, the films, the press, and the events that took place in the United States in support of the
\end{abstract}


Soviet Union, this article has two objectives: 1) to describe the context of that cultural production and verify that what Carpentier account is not the result of the invention of an ingenious novelist, but part of a forgotten history that the novel rescues; 2 ) explore the possibilities of literature to bring up an "other" memory and to articulate a new reading on the relations between the United States and the Soviet Union that challenges the official historical account and exposes the relationship between narration and power.

Keywords: Alejo Carpentier; La consagración de la primavera; russophilia; Second World War; propaganda.

\section{Resumen}

La novela La consagración de la primavera de Alejo Carpentier describe la opinión favorable del pueblo estadounidense sobre la Unión Soviética durante la Segunda Guerra Mundial como un fenómeno de "euforia rusófila". Este artículo tiene como objetivo partir de este concepto que la novela presenta para, como un trapero benjaminiano, redescubrir los residuos históricos que han quedado en los márgenes de la historia oficial. Revisando el contexto cultural, los libros, las películas, la prensa, y los eventos que se celebraron en Estados Unidos en apoyo a la Unión Soviética, este artículo persigue dos objetivos: 1) describir el conjunto de esa producción cultural y comprobar que lo que Carpentier cuenta no es fruto de la invención de un novelista ingenioso, sino parte de una historia olvidada que la novela rescata; 2) explorar las posibilidades de la literatura para hacer emerger una memoria "otra" y para articular una nueva lectura sobre las relaciones entre Estados Unidos y la Unión Soviética que desafíe el relato histórico oficial y ponga al descubierto la relación entre narración y poder.

Palabras clave: Alejo Carpentier; La consagración de la primavera; rusofilia; Segunda Guerra Mundial; propaganda. 


\section{"Esa euforia rusófila"}

Enero, 1943. Enrique aterriza en Nueva York. Después de pasar un largo periodo en Europa, que le lleva a conocer de primera mano los horrores del nazismo y la guerra, Enrique decide regresar a Cuba, su país natal, mientras contempla el "ocaso de Europa". Una vez instalado en La Habana se entera por la prensa de que la Segunda Guerra Mundial ha comenzado. A diferencia de lo que sucede en la Guerra Civil española, donde vive y cuenta en primera persona el conflicto, la Segunda Guerra Mundial la observa desde la distancia, desde la protección que le concede un océano de por medio, que le mantiene ahora alejado de las bombas y de las trincheras, primero en La Habana y después en Nueva York. La distancia sustituye el testimonio por la propaganda y la visión directa por la construcción simbólica de la guerra. "Aquí, por la distancia, la guerra se traducía en imágenes”, dice Enrique nada más pisar Nueva York en La consagración de la primavera de Alejo Carpentier (553).

En los subcapítulos 22 y 23 del capítulo IV de La consagración de la primavera, Alejo Carpentier describe, con el rigor y la minuciosidad a la que nos tiene acostumbrados el narrador cubano, cómo se vivió la Segunda Guerra Mundial en Estados Unidos, a través de las impresiones que extrae Enrique, su protagonista, durante su estancia de dos meses en Nueva York. Enrique reconoce que en Nueva York "me sentí agarrado por una cierta 'atmósfera de guerra'” (553), debido a la euforia de un pueblo -el pueblo norteamericanoque vivía intensamente la guerra mediada por la prensa y las imágenes que llegaban a los cines y los carteles. Para definir esa "atmósfera de guerra", Enrique/Carpentier emplea un sintagma sin duda sorprendente: "esa euforia rusófila" (563), que podríamos definir como la existencia de una corriente de opinión mayoritaria que se muestra muy favorable al rol desempeñado por la Unión Soviética en la Segunda Guerra Mundial, o como la existencia de un imaginario que concibe al pueblo ruso como un pueblo honrado, noble y trabajador, valiente y heroico, que está dando su vida, incluso pagando con la 
muerte, en la defensa de aquellos valores que se encuentran en seria amenaza a causa del peligro que supone el nazismo y su política de guerra expansionista. En efecto, como Enrique señala, los valores de occidente -la vida y la libertad"se estaban defendiendo en Stalingrado" (552), y así lo propagaban los medios de comunicación estadounidenses. Enrique descubre en Nueva York la simpatía que despierta Rusia entre el pueblo americano. Obsérvese que no se dice "Unión Soviética”, sino "Rusia", como fórmula eufemística:

...y digo [euforia] "rusófila" y no "sovietófila", ya que las estaciones de radio de aquí eran más dadas a hablar de Rusia que de la Unión Soviética porque, en fin, Rusia era de hoy y de ayer, la permanente: la de Chostakovitch pero también la de Tchaikovski; la de Cholojof, pero también la de Tolstoi y Pushkin, a la vez que decir URSS hubiera impuesto a los locutores el reiterado trabalenguas de: IUAR-IS-IS, con la ambigüedad fonética de un YOU ARE que se prestaba a chistes. ${ }^{2}$ (563-4)

Stalingrado fue el punto de inflexión. El 25 de agosto de 1942 se declaró el estado de sitio en Stalingrado. Las fuerzas soviéticas fueron obligadas a retroceder a las afueras de Stalingrado ante el rápido avance de los panzer alemanes (Beevor 501). El 2 de febrero de 1943 el ejército alemán, compuesto por más de 300.000 hombres, fue derrotado en Stalingrado por el Ejército Rojo tras una larga batalla. Como se apunta en La consagración de la primavera:

...a medidados de enero, las gentes empezaron a maravillarse ante aquella increíble Batalla de Stalingrado (ya escrita en singular, con B mayúscula), que se venía prolongando desde hacía varias semanas y donde era evidente que los nazis no alcanzaban ventaja alguna. Y la idea de que esa Batalla se estaba constituyendo en el acontecimiento mayor de la Guerra, alentaba una creciente y esperanzada expectación en las gentes, expectación que desembocó en un amor "coast to coast" hacia la Unión Soviética. (556-7) 
La victoria soviética en la batalla de Stalingrado constituye, en efecto, el momento de mayor euforia rusófila:

Un calendario con vista de la bahía de Nápoles [Enrique se encuentra en el restaurante italiano Gran Ticino de Nueva York] marcaba la fecha del 2 de febrero. Y no había acabado de almorzar cuando los radios, los altavoces, las ediciones especiales, anunciaron la derrota de los alemanes en Stalingrado, la capitulación de Von Paulus, el desplome (iprimero en esta guerra!) de todo un ejército nazi. Y fue el entusiasmo, y fue la grita y fue el júbilo. Y, ese día, el éter se llenó de ondas que, sobre los cielos turbios y revueltos de New York, hicieron sonar las campanas de La gran pascua rusa, y los acordes macizos y triunfales de La gran puerta de los boyardos de Moussorgsky. (563)

La euforia rusófila, la expectación que desemboca en amor, se registra en periódicos, revistas, en las carteleras de los cines y en las canciones que suenan en la radio. Rusia se vuelve omnipresente y su presencia es siempre positiva:

Ahora, a todas horas, sonaban Grandes pascuas rusas, danza del Príncipe Igor, Obertura 1812, Rusias de Balakirew, batallas de La ciudad invisible de Kitèje, dumkas y balalaikas, coros cosacos, gopaks y trepakcs, músicas de César Cui, de Glinka, de Liadow, y sobre todo, de Chostakovitch, apareado a Tchaikovski en todos los aparatos de radio del país. (557)

Y añade:

Muy de prisa, luchando contra el reloj, y con enormes despilfarros de bicarbonato en los estudios para nevar falsas calles, techos de isbas y cúpulas periformes, había sacado ya Hollywood una primera película (...) donde se exaltaba el heroísmo del pueblo soviético. El acorazado Potemkine [sic], Octubre, Tchapaieff, La línea general, aparecieron en las carteleras de varios cines. Boris Godunof fue coronado nuevamente en el Metropolitan. (557) 
De igual modo, Enrique observa "en una edición de Life, las filas de prisioneros alemanes macilentos, maltrechos, de cabezas vendadas, de brazos en cabestrillo, sacados por soldados soviéticos de las ruinas de Stalingrado" (564), pero también contempla horrorizado cómo La Internacional se ha convertido en un baile en el cabaré Rainbow Room, frecuentado por la alta sociedad neoyorquina (568-9). La Unión Soviética -sus iconos, sus símbolos, sus películas, sus canciones- se ha integrado plenamente en la vida cotidiana del pueblo norteamericano, que ahora siente admiración -"esa euforia rusófila"por quienes habían representado (y representarán en la Guerra Fría) un modelo de vida y de sociedad antagónico del que defiende el modelo capitalista de Estados Unidos.

Si se tratara de otro escritor, podríamos llegar a especular si acaso no estamos ante una invención de un novelista ingenioso; pero teniendo en cuenta que estamos hablando de Alejo Carpentier, un autor que documenta con rigor y exhaustividad todo lo que escribe, nos vemos abocados a pensar que lo que la novela describe es históricamente cierto. Como señala Julio Rodríguez Puértolas, Carpentier "se documenta hasta el detalle" con "afán de exactitud y rigurosidad (de verosimilitud)" (45). Por su parte, Roberto González Echevarría apunta que "la historia está presente en la narrativa de Carpentier de forma rigurosa; no evocan sus novelas un pasado creando un vago 'aire de época', sino mediante una sólida documentación y una impecable y a veces alucinante fidelidad cronológica" (203). Carpentier, que reivindicaba la tarea del novelista como la del "cronista de Indias" ("La novela latinoamericana..." 48), hizo de la literatura un documento para narrar la historia y un lugar de memoria donde los acontecimientos que el relato oficial secuestra y borra pudieran permanecer inalterables con el paso del tiempo. Pero el objetivo de este artículo no es únicamente verificar, como si la labor del historiador se redujera simplemente a la de un notario de la realidad, si lo que Carpentier ha escrito en su novela es verdadero o no, o evaluar la solvencia del escritor como 
documentalista; se trata también -o más bien- de articular y de hacer posible una nueva lectura acerca de las relaciones entre Estados Unidos y la Unión Soviética durante la Segunda Guerra Mundial, y de desafiar, con ella, el modo en que se ha construido un relato histórico que ha prescindido de una parte, sin duda relevante, de esa misma historia. Se trata de recuperar una parte del acontecimiento borrado para producir un nuevo sentido de la historia.

Volver al capítulo IV de La consagración de la primavera nos permite constatar que la historia fue de una forma distinta a como esta ha sido contada, pero también nos permite imaginar que, en cierto momento de la historia, siempre contingente, los acontecimientos podrían haberse desarrollado de otro modo. No se trata de pensar y leer La consagración de la primavera solamente como un documento histórico que nos permita extraer un conocimiento de la historia, sino como un texto que, al poner ante nosotros un nuevo conocimiento -o mejor: al permitirnos redescubrir un conocimiento secuestrado-, nos impulsa a exhumar imaginarios históricos olvidados para actualizar unas condiciones de posibilidad para una historia distinta. El relato histórico que se configuró durante la Guerra Fría hace pasar por inverosímil la existencia de un periodo histórico en que ambas potencias y ambos modelos económicos, irreconciliables según el relato oficial, pudieron cooperar y sentir admiración mutua, un momento histórico que dio lugar a una inmensa producción cultural en la que "esa euforia rusófila" quedó registrada. Para establecer este relato se borró este breve paréntesis que describe -y en cierto modo rescata- Carpentier en su novela. El relato de la Guerra Fría prescindió de este paréntesis para actualizar el anticomunismo estadounidense anterior a la Segunda Guerra Mundial y para así nutrir el anticomunismo posterior a la guerra, que tuvo su punto álgido en el macartismo, entre 1950 y 1956. Esta novela de Alejo Carpentier, al conferirle de nuevo existencia a ese fenómeno cultural borrado, no solo le devuelve la historicidad -este episodio ha existido realmente- sino que evidencia que la historia, o su relato, se escribe en función 
de intereses concretos, y, en consecuencia, el cuestionamiento del relato dominante supone así mismo un desafío a aquellos que lo han construido para mantener su hegemonía y su régimen de verdad.

El objetivo de este artículo es reconstruir, a partir de los subcapítulos 22 y 23 del capítulo IV de La consagración de la primavera de Alejo Carpentier, la "estructura de sentimiento" -siguiendo la terminología de Raymond Williams (174-85) - de una época concreta y en un lugar específico, contrastando con datos estadísticos lo que el novelista cubano referenció en su novela, pero también con la producción cultural del tiempo histórico que la novela describe. Para eso, y acaso como un trapero benjaminiano, traté de encontrar y recoger, en los márgenes del relato oficial, los residuos históricos que ese mismo relato oficial había desechado para constituirse. Como en El libro de los Pasajes de Walter Benjamin, la memoria solo se puede rescatar descubriéndola entre "harapos y desechos" (739). Cuando el historiador se sitúa en los márgenes y observa lo que ha quedado fuera del relato, es entonces capaz de entender el modo en que los vencedores escriben la historia, y de descubrir que en los residuos, en los episodios históricos de los que el relato oficial prescinde, se encuentra el sentido que vincula narración y poder, que en la borradura de un periodo de la historia se halla la explicación de la permanencia de las clases dirigentes en el poder. Para llevar a cabo esta tarea, aproveché una estancia de investigación en la New York University para, durante dos meses, tratar de revivir aquellos dos meses -enero y febrero-de 1943 en los que el protagonista de La consagración de la primavera permaneció en Nueva York. Seguir los pasos del personaje para, una vez recorridos de nuevo, poder captar esa misma atmósfera, esa misma "euforia rusófila". Leer los libros que Enrique pudo leer, asistir a los lugares a los que asistió, ver las películas que se anunciaban en las carteleras del Nueva York en enero y febrero de 1943, leer los periódicos de aquellos días; en definitiva, poder volver a ese momento de la historia en que los hechos se produjeron de una manera otra. 


\section{Las encuestas y el estado de opinión}

El primer paso para comprobar la existencia de esa "euforia rusófila" de la que nos habla Carpentier en su novela se ha de dar en la revisión de las encuestas realizadas en Estados Unidos durante los años que duró la Segunda Guerra Mundial.

En su desmitificador ensayo American Opinion and the Russian Alliance (19391945), Ralph B. Levering saca a colación unas encuestas realizadas en julio de 1941. A la pregunta "In the present war between Germany and Russia, which side would you like to see win?", el $72 \%$ de los encuestados respondió que prefería la victoria de los rusos frente al $4 \%$ que se descantaba por la victoria alemana; sin embargo, su preferencia no iba acompañada de optimismo: el $47 \%$ creía que los alemanes se iban a imponer a los rusos, en cuya victoria solo confiaba un $22 \%$ de los encuestados. Del mismo modo, los resultados de la encuesta mostraban que "the typical response of the members of the sample as follows: 'Russia is not imperialistic, but Germany is. Russia, even if she won, would not invade the United States, whereas Germany probably would"” (Levering 43).

En su artículo "American Attitudes toward Russia", publicado apenas dos años después de terminada la guerra, Warren B. Walsh señalaba que, si bien en un primer momento "[f]or some time the majority of Americans felt that nazism, as typified by Germany, was less bad than communism as typified by Russia” (184), "[o]ur sympathies, however, swung sharply in favor of Russia after Germany invaded the USSR. When asked which side we wanted to see victorious we picked Russia three to one, although some twenty-two million people said they didn't have a preference. The Red Army was much more popular with us than the Soviet government" (185). El historiador belga Jacques R. Pauwels en El mito de la guerra buena detalla cómo buena parte del capital financiero de los Estados Unidos y empresas como Esso, Ford, JP Morgan, Kodak o General Motors colaboraron estrechamente con Hitler durante los años 30 
y 40. Para el capital financiero, el fascismo constituía un peligro menor que el que reperesentaba el comunismo para sus intereses, a la vez que recibían algunas de sus políticas -como la prohibición de los sindicatos- como muy positivas para la concepción de sociedad que ellos tenían $(36,39,48,48)$. De hecho, estos sectores de la sociedad, cuando se inicia la cooperación entre Estados Unidos y la Unión Soviética, para enfrentarse al enemigo común que constituía el nazismo, consideraron que Estados Unidos había elegido el "enemigo equivocado" (193). Como apunta Chris Bambery, "entre las clases dirigentes existía un grupo que no percibía a Hitler como una amenaza” (55) y, en junio de 1941, Harry S. Truman, a la sazón vicepresidente de Franklin D. Roosevelt, declaró ante la prensa: "Si vemos que Alemania, gana, debemos ayudar a Rusia; si gana Rusia, tendremos que ayudar a Alemania y, de ese modo, dejar que se maten cuantos más mejor" (cit. en Bambert 55). Fue la batalla de Stalingrado, como hemos visto en La consagración de la primavera, la que impulsó el cambio en la "estructura de sentimiento" en Estados Unidos. Según Walsh, a mediados de 1943, casi 73 millones de estadounidenses estaban a favor de una asociación igualitaria con Rusia en la guerra y en la paz (185).

Del mismo modo, en su libro Aid to Russia, 1941-1946: Strategy, Diplomacy, the Origins of the Cold War, George C. Herring Jr. apunta que el respaldo popular a la Unión Soviética aumentó significativamente entre julio y agosto de 1941. Una encuesta de Gallup del 5 de agosto de 1941 -señala Herring- mostraba que el $70 \%$ de los encuestados estaba a favor de la venta de suministros de guerra a la URSS, y otra encuesta, realizada en septiembre, revelaba que el 49\% aprobaba la concesión de créditos (18). Incluso, según Herring, un bastión anticomunista como la American Federation of Labor, respaldó la provisión de suministros para derrotar la "Nazi war machine" (18).

Por su parte, Jacques R. Pauwels muestra cómo "esa euforia rusófila" incluso se mantuvo cuando las circunstancias geopolíticas ya habían cambiado. Dice Pauwels que "en la primavera de 1945 todavía el aliado soviético contaba 
con el aprecio del pueblo americano, así como de los soldados americanos estacionados en Europa" (168-9). Una nueva encuesta Gallup, de marzo de 1945, reveló que el 55\% de los americanos se posicionaba a favor de que su país siguiera manteniendo a la URSS como aliado tras el fin de la guerra. La encuesta también mostraba que los soldados americanos "sentían por sus camaradas del Ejército Rojo, casi sin excepción, una mezcla de simpatía, admiración y respeto" (169).

Sin embargo, conviene así mismo señalar, siguiendo a Melvin Small, que las encuestas solamente ofrecen una parte de la historia. En su artículo de transparente título, "How We Learned to Love the Russians", Small plantea que, para tener un perfil completo del estado de opinión hacia la Unión Soviética en Estados Unidos, resulta imprescindible observar el modo en que se construye la opinión pública. Para alcanzar una respuesta satisfactoria, Small revisa la presencia de la Unión Soviética en revistas, periódicos, ensayos, películas y reportajes de guerra en Estados Unidos. En este sentido, el artículo de Melvin Small resulta de consulta fundamental. En primer lugar, Small recoge los datos de la presencia de la Unión Soviética en los medios de comunicación americanos y, a partir de ellos, elabora unas conclusiones sin duda significativas. Small sostiene que, durante los años de la guerra, las revistas estadounidenses dieron un giro notable en su manera de tratar a la Unión Soviética. Small recogió 795 artículos de 23 publicaciones distintas y, para evaluarlos, dividió en tres periodos los años de la guerra: de agosto de 1939 a junio de 1940, de julio de 1940 a junio de 1941, y de julio de 1941 a diciembre de 1944. Luego, confeccionó unas tablas que muestran que, en el tercer periodo, que es el que ahora nos interesa, Rusia fue tratada de forma más favorable durante el tercer período que durante los dos primeros (459); incluso los medios más conservadores aprobaron la labor de los rusos en la guerra y se redujeron -si bien nunca llegaron a desaparecer- los artículos abiertamente antisoviéticos. 
En una de las tablas que presenta Small se observa la línea ascendente que registra la opinión favorable hacia la Unión Soviética en todo el espectro político -en revistas tanto conservadoras como liberales o moderadas- en el tercer periodo de la Segunda Guerra Mundial. Si en el primer periodo de la guerra solamente se publicaron 13 artículos favorables sobre la URSS en la prensa liberal, uno en las revistas moderadas y tres en las más conservadoras, en el tercer periodo los artículos favorables se multiplicaron por 13 en las revistas liberales, Ilegando a alcanzar un total de 74 (el 55\% del total), al tiempo que se publicaron 95 artículos favorales en las revistas moderadas (el 50\% del total) y hasta 50 en las conservadoras (alcanzado el nada desdeñable porcentaje del $33 \%$ del total, siendo el $41 \%$ artículos negativos y el $23 \%$ neutrales).

Por lo que respecta a la prensa diaria, Small subraya, tras analizar sus editoriales, que las victorias soviéticas de 1943 llevaron a los editores a adoptar un punto de vista sobre la Unión Soviética "sorprendentemente benévolo" (462). De hecho, a finales de 1944, según Small, solamente cabeceras como New York Daily News, el Chicago Tribune y el Wall Street Journal podían considerarse antisoviéticas, e incluso periódicos como el Kansas City Star y el Detroit News, bastiones del "Middle American thought", ofrecían opiniones más favorables que desfavorables sobre la URSS (462). Parece indiscutible la alta presencia que tuvo, en aquellos años, la Unión Soviética en la prensa norteamericana. Según los datos que ofrece Levering, solo durante la última semana de junio de 1941, la guerra entre la Unión Soviética y la Alemania nazi, así como las relaciones entre Estados Unidos y la Unión Soviética, ocuparon el 72,3\% de las portadas de los periódicos norteamericanos; esta cifra se redujo drásticamente solo un mes después, ocupando estos temas un $21,7 \%$ de las portadas, pero, en opinión de Levering, incluso este porcentaje, claramente inferior, "represents several thousand words of news each week on the page most likely to be read" (Levering 54). 
Pero más allá de los datos, que son sin duda elocuentes, resulta así mismo pertinente revisar -para obtener una visión más detallada del fenómeno- el contenido de esos artículos. Levering, en su libro, ofrece una descripción exhaustiva de cómo fue retratado el pueblo ruso en las revistas norteamericanas:

Life carried picture stories favorable to Russia with such concrete titles as "Russian Courage and Cold Rout Nazis" and "Russian Parents Lament Dead Son at Scene of German Execution." Time selected President Roosvelt as its man of the year for 1941 because of his foresight in supporting preparedness and aid to the Allies, and underlined the importance of unity by placing Stalin to Roosevelt's left and Churchill to his right on the cover of the 5 January 1942 issue. In the lead article Stalin was recognized as "the only leader who has yet to face a major German drive without a militar disaster," and Roosevelt was hailed as the "leader of the democracies against Hitler." Such recognition of Stalin as Rooselvet's partner in Allied leadership redounded to Russia's benefit among Americans. Of more specific assistance were the several Russian military leaders and strategists introduced to the public on the convers of Time during the year. (70)

Como se observa, no era solamente el "pueblo ruso" -categoría más neutral y despolitizada- la que acaparaba la atención en Estados Unidos, sino también el propio Stalin, retratado como un político que reunía todas las cualidades que un gran líder debe tener: "Stalin has all the qualities of the great fighting leader", dijo Averell Harriman en la radio CBS en 1942, añadiendo después: "He has confidence in the Red Army and the Russian people, and they have implicit confidence in him. He knows that Hitler will be destroyed and that the Red Army will play a leading role in the glory of this achievement. But he looks upon the war against Hiler as a united effort with Britain and the United States." (cit. en Levering 104-5). Según Levering: 
The culmination of the pro-Russian blitz in the fall of 1942 was the benign, almost saintly painting of Joseph Stalin with the snowy Russian winter as backdrop which appeared on the cover of Time's New Year's issue. Stalin had been man of the year for 1939 as well, but what a different man he was now! In 1939 he had been Hitler's partner in evil, the shrewd, ruthless Bolshevik whose moves had thrown the world into war. Now he had almost supernatural wisdom and understanding. "Only Joseph Stalin fully knew how close Russia stood to defeat in 1942," Time said with genuine awe, "and only Joseph Stalin fully knew how he brought Russia through." Stalin's acievements were unequaled in the modern era, Time implied: "He collectivized the farms and built Russia into one of the four great industrial powers on earth." "The U.S., of all nations, should have been the first to understand Russia," the article stated in reference to the similarities between the two nations. "Ignorance of Russia and suspicion of Stalin were the two things that prevented it." "As Allies fighting the common enemy, the Russians have fought the best fight so far," Time concluded. "As post-war collaborators, they hold many of the keys to a successful peace." (105)

En breve: "Stalin himself, Time remarked, once regarded as a 'sort of unwashed Genghis Khan with blood dripping from his fingertips', had come to see 'increasingly benign,' even a 'nice old gentleman'” (Herring 22). La descripción de Stalin de Time se complementa con la fotografía que ilustra la portada del número en la que anuncia a Stalin como "man of the year" en 1942.

\section{Cine y espectáculos}

No solo la prensa escrita favoreció ese clima de opinión; también Hollywood participó activamente en generar "esa euforia rusófila" de la que hablaba Carpentier. De hecho, veíamos arriba que La consagración de la primavera narra cómo la industria del cine empezó a improvisar decorados nevados para representar escenas bélicas en las que narrar las heroicas gestas del ejército y del pueblo ruso. 


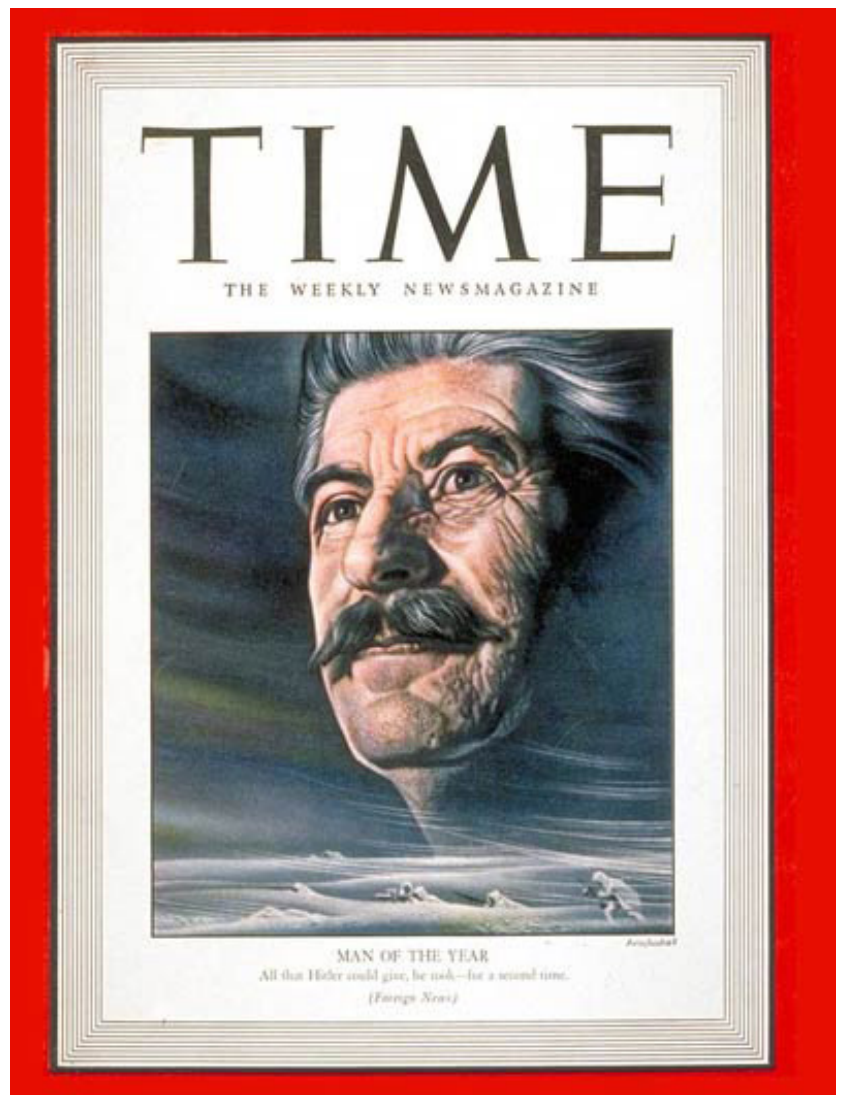

Time, 4 de enero de 1943

Small cuenta en su artículo ya citado que la imagen de la URSS en el cine norteamericano cambió radicalmente en 1942. Fue entonces cuando se descolgaron de las carteleras las películas satíricas y antisoviéticas -como Ninotchka, Comrade X, Public Deb Number One o He Stayed for Breakfast-, para dejar su espacio a reportajes sobre las heroicas batallas del Ejército Rojo, documentales producidos en la Unión Soviética y narrados por respetadas 
personalidades estadounidenses, o películas producidas en Hollywood con un fuerte componente épico y protagonizadas también por las estrellas de cine del momento, como Walter Brennan, Robert Taylor, Walter Huston, Gregory Peck y Susan Peters, que mostraban la valentía y la heroicidad del pueblo ruso en su lucha contra el nazismo. Entre estas películas Small menciona Mission to Moscow, The North Star, y Song of Russia. Estas películas, además, tuvieron un enorme éxito de taquilla (Small 460-1). Levering añade a la relación de títulos presentada por Small otros títulos como The Siege of Leningrad, The City That Stopped Hitler-Heroic Stalingrad, We Will Come Back, o Day After Day (Levering 123). Pero, en la opinion de Levering, la película que constituye la culminación de esa campaña de amistad entre Estados Unidos y la Unión Soviética -y que promueve esa "euforia rusófila"- es The Battle of Russia:

Beginning in 1943, this extremely favorable portrait of a courageous ally was shown to each of the several million Americans who entered the services. With equal courage and willingness to sacrifice, the film implied strongly, Hitler would soon be doomed. It was a patch-work movie, bringing together highlights from several previous movies about Russian resistance. The Battle of Russia, which was released for commercial showing in the fall of 1943, was in many respects the culmination of the official and private campaigns in this country for friendship toward Russia. (123)

Pero no menos significativas son Mission to Moscow, y North Star. La primera, filmada en 1943 y dirigida por Michael Curtiz, está basada en el diario de Joseph E. Davies, embajador de los Estados Unidos en la Unión Soviética entre 1936 y 1938 -de quien más adelante volveremos a hablar. La versión cinematográfica del libro de Davies está protagonizada por un empresario norteamericano que es nombrado embajador de Estados Unidos ante la Unión Soviética por el presidente Roosevelt, quien le encomienda la misión de indagar las verdaderas intenciones de la URSS en la Segunda Guerra Mundial. A través de los ojos 
del embajador, la película de Curtiz no solo ensalza a un pueblo laborioso que trabaja por el bien común, sino que también pone en valor el milagro económico soviético e, incluso, se describen las denominadas purgas de Stalin como juicios justos. Mission to Moscow constituye un auténtico retrato propagandístico de la Unión Soviética, curiosamente diseñado por Hollywood. Por su parte, North Star, de Lewis Milestone, también producida en 1943, narra la heroicidad y la hazaña de unos jóvenes rusos que se ven obligados a abandonar su vida sencilla para combatir a Hitler, una vez el dictador nazi ha puesto en marcha su "Operación Barbarroja" contra la URSS.

El cine no fue el único espectáculo que sirvió para modificar la opinión del pueblo norteamericano. Paul Willen, en un artículo titulado "Who ‘Collaborated' with Russia?”, publicado en 1954, describe el papel destacado que representó el Congress of American-Soviet Friendship en la organización de importantes eventos que tenían por objetivo la rendición de un tributo al pueblo ruso por la extraordinaria labor que estaba realizando en el frente de batalla. Willen destaca, entre los múltiples eventos organizados por este Congreso, un encuentro celebrado en 1943, que se organizó para conmemorar el aniversario de la Revolución de Octubre, y así mismo la multitudinaria manifestación que tuvo lugar en el Madison Square de Nueva York el 22 de julio de 1942, unos meses antes de la llegada de Enrique en La consagración de la primavera.

A juzgar por los ensayos citados, parece que existió "esa euforia rusófila" en las calles de Nueva York. Para corroborar la veracidad histórica de esta propuesta literaria de Carpentier, tanto la lectura de fuentes primarias como el estudio de la prensa de la época -la misma que Enrique leería en su estancia en Nueva York- permiten calibrar la verdadera dimensión de "esa euforia rusófila" de la que hablaba Carpentier y que los ensayos consultados y hasta el momento referidos parecían verificar. Una lectura atenta de las páginas de cultura y espectáculos del New York Times de enero y febrero de 1943, meses 
en los que dura la estancia de Enrique en Nueva York en La consagración de la primavera, nos permite advertir que, en efecto, se sucedían eventos con temática rusa o soviética en Nueva York. Por ejemplo, en la edición del 7 de enero de 1943 ("Concert Committee Head"), se anuncia que el famoso Carnegie Hall de Manhattan iba a acoger, el 23 de enero, un concierto del celebérrimo compositor y pianista de jazz Duke Ellington, auspiciado por el no menos célebre Orson Welles, para recaudar fondos con los que ayudar a la Unión Soviética. Del mismo modo, se anuncian en las páginas del New York Times las nuevas producciones cinematográficas que sobre Rusia se iban a filmar: se habla, por ejemplo, de la película This is Russia de Casey Robinson, que finalmente se estrenaría bajo el título de Days of Glory en 1944 ("By Telephone to"), y así mismo comprobamos que había varias películas soviéticas de temática revolucionaria en cartera simultáneamente: Moscow Strikes Back, Fortress on the Volga, y Ten Days That Shook the World ("Best War Fact Film Opens”). También New York Times recoge noticias curiosas sobre la vida cotidiana en la URSS -acaso para espantar el fantasma del comunismo entre sus lectores y tratar de identificar valores comunes y de este modo reducir aquello que les distanciaba del otro- como un artículo sobre las navidades en Rusia, que celebran incluso los no creyentes, publicado el 7 de enero de 1943 ("Christmas Eve in Russia"). Sin duda contribuyeron a "esa euforia rusófila" artículos como el publicado por el músico soviético Sergei Prokofieff sobre la admiración mutua y el intercambio cultural que se estaba produciendo entre el pueblo ruso y el pueblo norteamericano, publicado el 2 de enero de 1943 (“Prokofieff, Soviet Composer"). La lectura de la prensa de la época evidencia que, en efecto, la presencia de la URSS era constante en la vida norteamericana en enero y febrero de 1943. 


\section{Libros}

En la vida, pero también en las librerías. No fueron pocos los libros que durante estos años se publicaron sobre la Unión Soviética en Estados Unidos, algunos para tratar de ofrecer explicaciones sobre el verdadero funcionamiento del Estado socialista nacido con el triunfo de la Revolución, otros para abiertamente elogiarlo, incluso justificando o edulcorando los aspectos más controvertidos del estalinismo. Resulta paradigmático el libro The Russians de Albert Rhys Williams, publicado en 1943 y reseñado en New York Times el 10 de enero de 1943. Aunque resulta imposible ofrecer, por una cuestión de espacio, un análisis detenido del libro de Williams, que sin duda lo merecería, sí es preciso mostrar de forma detallada algunos aspectos del mismo que consideramos relevantes. El libro describe, de forma minuciosa, los distintos aspectos relativos a la guerra, desde el funcionamiento de la guerrillas (Williams 62-4) hasta la propaganda y la cultura en el frente $(60,66)$, o las virtudes de un pueblo que se encuentra en todo momento preparado para la defensa (66); pero también otros aspectos que tienen que ver con el funcionamiento del Estado y la sociedad soviética: el sufragio universal (85-7), los logros de los planes quinquenales (101-3), la agricultura (108-13), la industria (120-1), los sindicatos (142-3), la mujer (167-8), la educación (183), la salud (187), la prensa (202-3), la religión (209) o la cuestión del socialismo en un solo país (221-2). Si bien no es posible mostrar fragmentos sobre todas las cuestiones citadas, no podemos sino dejar de reproducir un fragmento que se encuentra al comienzo de The Russians en el que su autor anota los diez logros más encomiables de la Revolución; el fragmento, aunque largo, merece ser reproducido por entero, ya que nos permite captar, de un solo vistazo, el estilo y el objetivo del libro: 
What is it that the Revolution has given him to fight for? Or rather, what has he achieved for himself through the Revolution?

First- the 250,000 collective farms equipped with modern machinery and power, in which all members have a stake and share.

Second- the practical disappearance of racial and national antagonism among Jews, Russians, Tartars, Armenians, and 185 other peoples now enjoying equal rights and privileges.

Third- an ever-expanding economy, creating a ceaseless demand for more and more technicians, foremen, engineers, chemists, architects, teachers, journalists, physicians.

Fourth- the extensive system of schools, colleges, technicums, training the youth for these positions. Teaching 50 million illiterates to read and write; publishing over 30,000 new book titles each year.

Fifth- the emancipation of women from old sex taboos and disabilities of the past -all positions and professions now open to them on the same terms as men; establishing a nation-wide system of nurseries and kindergartens.

Sixth- the practical elimination of those scourges of cholera, smallpox, and typhus that once ravaged the country.

Seventh- abolition of the unemployment, with the right of every citizen to work, education, and leisure written into the Constitution.

Eight- the system of insurance against accident, illness, and old age, liberating the people from the fear and dread of want.

Ninth- the elimination of crises and depressions by striking a balance between production and consumption-putting the money into the pockets of the people to buy back the good that they make as fast as they can make them. Tenth-a system of planning, working toward on ordered, wasteless development of the nation's resources. (5-6)

En The Russians, Williams ofrece así mismo una descripción muy positiva de Stalin. Williams destaca, en primer lugar, su altura política (74) para, más adelante, subrayar el carácter sencillo y el estilo austero del líder soviético: 
That is the great simplicity of the man in private life and public address (...) Quite as unostentatious is he in dress, bearing, and manner of life. With his wife, the sister of Kaganovich, he lives in a modest three-room apartment in the Kremlin, and has a house in the country an hour away from Moscow. With simple, almost ascetic tastes, he prefers the classics, enjoys the company of old comrades who, with him, were unswerving in devotion to the cause from the beginning. (79)

Por otro lado, si bien señala que las purgas crearon consternación en la Unión Soviética (97) y que fueron "a massacre and madness" (97), también afirma que sin ellas la Unión Soviética se hubiera encontrado en una situación de permanente debilidad con la que no hubiera sido capaz de resitir la infiltración y la invasión nazi (97-8).

Más o menos en la misma línea se encuentra el libro The Truth about Soviet Russia, publicado en 1942 por Sidney Webb. El libro contiene secciones donde el autor se interroga sobre algunas cuestiones que, en su opinión, conviene aclarar para evitar malentendidos o despejar los prejuicios que existen en Estados Unidos acerca del sistema político soviético. Por ejemplo, se formulan las preguntas “Is Stalin a Dictator?" o "Is the USSR a Political Democracy?" La respuesta a la primera pregunta es la siguiente:

To answer the first question -Is Stalin a dictator?- we must agree on what meaning is to be attached to the term dictator: otherwise the argument is a waste of time. Assuming that we accept the primary meaning of the term dictator, as it is defined in the New English Dictionary -"a ruler or governor whose word is law; an absolute ruler of the state -and who authoritatively prescribes a course of action or dictates what is to be done" (the example given being the Dictators of ancient Rome) -Stalin is not a dictator. So far as Stalin is elated to the constitution of the USSR, as amended in 1936, he is a duly elected representative of one of the Moscow constituencies to the Supreme 
Soviet of the USSR. By this assembly he has been selected as one of the thirty members of the Presidium of the Supreme Soviet of the USSR, accountable to the representative assembly for all is activities. It is this Presidium which selects the Council of Commissars (Sovnarkom) and, during the intervals between the meetings of the Supreme Soviet, controls the policy of the Sovnarkom. (Webb 16)

Y añade:

In May 1941, Stalin hitherto content to be a member of the Presidium, alarmed at the menace of a victorious German army invading the Ukraine, took over, with the consent of the Presidium the office of Prime Minister and Minister of Defense (...); in exactly the same way, and for a similar reason -the world war- that Winston Churchill, with the consent of the House of the Commons, became Prime Minster and Minister of Defense with Chamberlain, the outgoing Prime Minister, as a prominent member of the British Cabinet (...) By declaring, in May 1941, a state of unlimited national emergency, President Roosevelt legally assumes a virtual dictatorship of the United States. He has power to take over transport, to commandeer the radio for the purposes of propaganda, to control imports and all exchange transactions, to requisition ships and to suspend laws governing working hours, and, most important of all, to decide on industrial priorities and, if necessary, to take over industrial plants. (16-8)

Stalin, pues, apunta Webb, si llevó a cabo una concentración de poderes lo hizo forzado por las circunstancias, del mismo modo que lo hizo su homólogo británico. La segunda cuestión la responde de la siguiente manera:

In answer to the second question -Is the USSR a political democracy?- it is clear that, tested by the Constitution of the Soviet Union as revised and enacted in 1939, the USSR is the most inclusive and equalized democracy in the world. The Supreme Soviet of the USSR consists of two chambers -the Soviet of the Union and the Soviet of Nationalities. The Soviet of the Union is directly elected 
by the citizens in electoral districts of one deputy for three hundred thousand inhabitants, the number of deputies today being over twelve hundred. The Soviet of Nationalities, with over six hundred deputies, also directly elected, aims at giving additional representation to ethnical groups whether manifested in colour or figure, language or literature, religion or manners, inhabiting large areas of the USSR. (19-20)

La promoción de una representación adicional a los grupos étnicos minoritarios le confiere al sistema soviético un grado democratizador del que carecen los sistemas parlamentarios occidentales. Webb enfatiza, a lo largo su ensayo, la igualdad racial que, a diferencia de Estados Unidos o Europa, ha alcanzado la Unión Soviética (23).

Otro libro interesante es One World de Wendell L. Willkie, publicado en 1943; y lo es no solo por su contenido, muy relacionado con los otros libros mostrados, sino también como objeto histórico, ya que su formato peculiar -folios sin prácticamente margen que contienen más de una página cada unoes consecuencia de la escasez de papel durante la guerra, lo que obligaba a los editores a inventar nuevos formatos de libro para ahorrar papel. ${ }^{3}$ En lo que concierne a su contenido, el libro cuenta, entre otras cosas, el viaje que realizó su autor a la República de Yakutsk, cuya visita le permitió contemplar el funcionamiento real del sistema soviético y destacar el papel central de la educación en la URSS (Willkie 38-39), así como las ventajas del sistema del Partido Único (40). Pero lo más interesante del libro acaso sea la narración de sus dos largos encuentros con Stalin, de tres y cinco horas cada uno. Un Stalin cansado, duro y sencillo aparece retratado en One World:

...he looked tired in September -not sick, as is so often reported, but desperately tired He had a right to be. He talks quietly, readily, and at times with a simple, moving eloquence (...) He has, I would say, a hard, tenacious, driving mind (...) Stalin is a hard man, perhaps even a cruel man, but a very able one. He has few illusions (...) On the personal side Stalin is a simple man, with no affectations 
or poses. He does not seek to impress by any artificial mannerisms. His sense of humor is a robust one, and he laughs readily at unsubtle jokes and repartee. $(33-4)$

Del mismo modo, el libro apacigua a sus lectores americanos asegurando que no hay que temer a la Unión Soviética, más bien, y al contrario, hay que apoyarla en su lucha contra el enemigo común, aprender de sus políticas y aprender a cooperar una vez termine la guerra:

Many among the democracies fear and mistrust Soviet Russia. They dread the inroads of an economic order that would be destructive of their own. Such fear is weakness. Russia is neither going to eat us nor seduce us. That is -and this is something for us to think about- that is, unless our democratic institutions and our free economy become so frail through abuse and failure in practice as to make us soft and vulnerable. The best answer to Communism is a living, vibrant, fearless democracy -economic, social, and political. All we need to do is to stand up and perform according to our professed ideals. Then those ideals will be safe. (35-6)

Y añade:

No, we do not need to fear Russia. We need to learn to work with her against our common enemy, Hitler. We need to learn to work with her in the world after the war. For Russia is a dynamic country, a vital new society, a force that cannot be bypassed in any future world. (36)

Otro libro peculiar es Shooting the Russian War, un reportaje fotográfico firmado por Margaret Bourke-White, publicado en 1942. La mirada de la fotógrafa puso el foco en la situación de la mujer en la Unión Soviética, que gracias a las políticas de igualdad impulsadas por el gobierno pudo convertirse en una ciudadana activa, en idéntico plano que los hombres. La presencia de 
la mujer como sujeto político activo acaso sea lo más destacable del libro, pero resulta así mismo interesante observar, como lo hemos hecho con otros libros, los argumentos de los que se sirve la autora para justificar las purgas como elemento garante de la unidad:

The unity of the Soviet people has been assured because whatever dissention existed during the last few years was wiped out. Thus no organized opposition is left. These drastic measures offer another explanation of why there is no fifthcolumn movement in the country -one secret of the Soviet Union's strength. But the all-sweeping corrective measures which were taken did leave a wake of fear. Even among the patriotic and loyal, this fear was noticeable (...). When I returned in the spring of 1941 I found that a crystallization had taken place. There was less of that refreshing experimentation with new things, and more conformity. No one could afford to make a mistake in ideology.

But in regard to this fear, the war was the great healer. With each month of the conflict, unity became more pronounced and dread lessened. Citizens had so many new tasks to do, tasks which demonstrated their loyalty, that they no longer had to fear whether their patriotism might be open to question. (17780).

También resulta oportuno, para medir la "euforia rusófila" de la autora, reseñar la presencia de Stalin en su libro. En un apartado titulado "I photograph Stalin" cuenta con todo detalle el momento en que fotografió a Stalin:

As I crawled on my hands and knees from one low camera angle to another, Stalin thought it was funny and started to laugh.

When his face lighted up with a smile, the change was miraculous. It was as though a second personality had come to the front, genial, cordial, and kindly. I pressed on through two more exposures, until I had the expression that I wanted.

I got ready to go, and threw my stuff back into the camera case; then I noticed a peculiar thing about Stalin's face. When the smile ended, it 
was as though a veil had been drawn over his features. Again he looked as if he had beenturned into granite, and I went away thinking that this was the strongest, most determined face I had ever seen. (217)

La fotografía fue portada del número del 29 de marzo de 1943 de la revista Life.

Otros libros de la época sobre la Unión Soviética, acaso menos apologéticos, pero igualmente importantes para aprehender la totalidad del fenómeno,

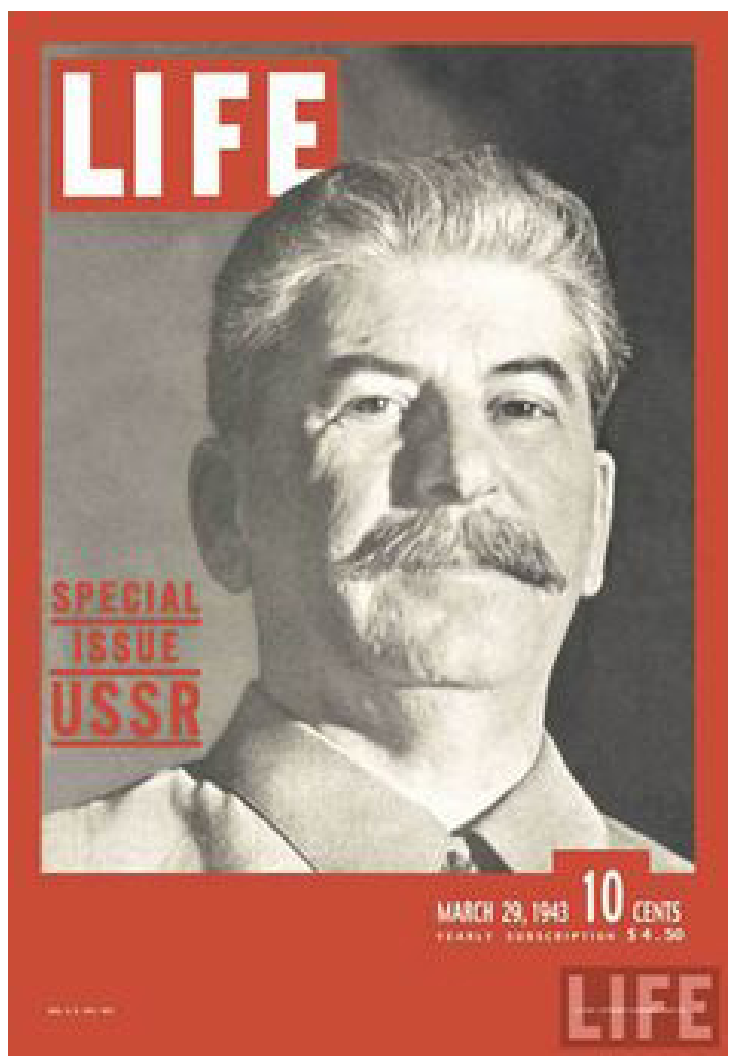

Life, 29 de marzo de 1943 
son American Opinion of Soviet Russia de Meno Lovenstein, publicado en 1941; Prelude to Vitory de James B. Reston, publicado en 1943, o más tardío, From A Political Diary. Russia, the Ukraine, and America (1905-1945) de Arnold D. Margolin, publicado ya en 1946. Tanto el libro de Lovenstein como el de Margolin se dedican a resaltar el nuevo rumbo, más moderado, que inició la Unión Soviética desde la promulgación de la Constitución de 1936, mientras que en el libro de Reston se pone el acento en la necesidad de cooperar con los rusos para vencer al enemigo común.

Pero no podemos terminar este repaso bibliográfico sin detenernos en la lectura de Mission to Moscow, el diario escrito por el embajador Joseph E. Davies en la Unión Soviética y publicado en 1941. El libro, si bien parte de la firme convicción anticomunista del autor, muestra todo lo que aprendió tras su paso por la URSS. De esta forma tan clara lo expone en la introducción:

I am equally firmly convinced that Communism, as such, cannot work on this earth, with human nature as it is (...) I am a firm believer in evolution as against revolution. I think our system is doing the best job for the common man and doing it more slowly but more surely because it seeks advance through evolution rather than though revolution.

Those convictions with which I went to Moscow, I brought back unchanged. I had, however, seen and learned much that I had not known, and I saw forces in being and apparently evolving which would have far-reaching effects on social and political conditions of the future. It renewed my faith in the Christian religion as indestructible and in the beneficences of our own form of government and our own way of life. (Davies xvi-xv)

Buena parte de las notas de su diario están dedicadas a los "Moscow trials" de febrero de 1937, y admite claramente que tuvieron un fin propagandístico (42), aunque del mismo modo afirma que las violentas purgas sirvieron para evitar un golpe de Estado (161) o la descomposición del ejército, que tras superar las adversidades se hizo más fuerte $(168,202-3)$. También destaca algunos de 
los avances más positivos que registró la Unión Soviética, como el desarrollo industrial o la promoción del trabajo público, aunque inmeditamente después saque de nuevo a colación el relato del terror estalinista (302-3).

En su libro Davies incluye una carta, fechada el 5 de junio de 1938, en la que cuenta el encuentro fortuito, pero muy agradable, que tuvo con Stalin, y donde le describe de forma muy positiva, como se puede ver en el siguiente extracto:

Upon the occasion of making my formal parting call upon President Kalinin and Premier Molotov on June 5, 1938, a very interesting situation developed.

When I was in Premier Molotov's apartment in the Kremlin, and within a very few moments after I had been seated, Mr. Stalin entered the room alone, came forward, greeted me very cordially, and he, Molotov, and myself engaged in discussions for two hours and fifteen minutes. Supplementing my telegram, I have the honor to report with reference thereto as follows:

After the usual preliminary amenities incident to the occasion of my call on the Premier by reason of my departure and transfer to Belgium, we entered upon a friendly and interesting talk. Stalin was particularly interested in President Roosevelt and asked many questions about him. He also referred in terms of much admiration to your Washington speech. (338-9)

Como curiosidad, diremos que se adjunta a la carta una fotografía dedicada de Stalin. Sus conclusiones, que están presentes a lo largo del libro, son claras y se pueden resumir en el siguiente párrafo:

Many fine things are being done under the present regime. Many noble enterprises have been projected which arouse sympathy and inspire intense admiration. No degree of altruism, humanitarian effort, or high and noble purpose, and no amount of material improvement in providing for things which elevate the standard of living of the proletariat, can ever compensate for the denial of freedom, of liberty, and the rights of the individual even as against the king. (308) 
Afirma también que "I do not think that the world is in any real danger from communism for many years to come" (317), precisamente porque "The Russia of Lenin and Trotsky -the Russia of the Bolshevik Revolution- no longer exists" (511). El libro de Davies es sorprendente en un sentido: a pesar de ser un convencido anticomunista muestra un retrato muy positivo de la URSS, si bien nunca deja de condenar -a diferencia de lo que ocurre en otros libros- las purgas.

\section{“Dramatic broadcast" y la construcción de la opinión}

Todos estos libros -salvo el publicado en 1946- pudo haberlos leído Enrique durante su estancia en Nueva York. Si nos hemos detenido a reseñarlos, es porque consideramos que sin duda tal producción bibliográfica contribuyó a la creación de ese estado de ánimo colectivo que en La consagración de la primavera se denomina "euforia rusófila".

Aunque buena parte de los libros y artículos publicados acerca de la Unión Soviética, como también las películas producidas o los eventos y las campañas organizadas para ayudar a la URSS, nacieron de una profunda y sincera admiración por el país de los soviets, es así mismo importante observar cómo "esa euforia rusófila" no fue solamente una reacción espontánea por parte del pueblo, sino que también estuvo diseñada "desde arriba": la construcción de esa opinión favorable respondía a una clara estrategia del gobierno para justificar su cooperación con quien hasta el momento había aparecido en el relato oficial como el mayor enemigo de los Estados Unidos y la democracia occidental: Ia Unión Soviética. El cambio de la política exterior y la diplomacia norteamericana no iba a ser aceptada por un pueblo que tenía una mirada prejuiciada y temorosa sobre todo aquello que concernía a la revolución y al comunismo. Era necesario modificar esa percepción para justificar el viraje diplomático y la cooperación con la Unión Soviética. El Estado puso entonces toda la maquinaria propagandística a trabajar -prensa, radio, cines, editoriales, 
etc.- para construir esa opinión favorable.

Todo empezó la noche del 22 de junio de 1941 con un discurso del primer ministro británico Winston $\mathrm{S}$. Churchill, que millones de americanos escucharon a través de la NBC. El discurso se pronunció cuando apenas habían pasado unas horas desde la invasión de Alemania y sus aliados a la Unión Soviética (Levering 39). Como afirma Albert Rhys Williams en The Russians, los americanos

...knew little about Soviet achievements; a great deal about Soviet follies, failures, cruelties. With this one-sided, distorted picture, no wonder Americans were amazed by the turn of events in Russia. They had come to believe that the country, hopelessly disorganized, was infested with Quislings and traitors (...). They had come to believe that the Soviets were on the road leading back to the Middle Ages and barbarism (...)They had come to believe that the Soviet people were cowed and spiritless or, seething with rebellion, were ready to rise up and welcome the invader. Then they hear Winston Churchill calling upon the British public to emulate the devotion and loyalty of the Soviet peoples to their leaders and government. They saw almost every Russian man, woman, and child working or fighting in defense of their soldiers are all vindicating that statement of Voroshilov, "Our soldiers are all the Soviet people." (4)

¿Qué dijo Churchill para modificar radicalmente la opinión de los americanos respecto a la Unión Soviética? Básicamente que la defensa de la integridad del Reino Unido y de Estados Unidos pasaba por la alianza y la cooperación con la Unión Soviética. Y lo hacía, acaso para despejar posibles sospechas ideológicas, apelando primero a su más firme anticomunismo:

No one has been a more consistent opponent of Communism than I have for the last twenty-five years. I will unsay no words that I have spoken about it. But all this fades away before the spectacle which is now unfolding. The past with its crimes, its follies its tragedies flashes way. (cit. en Levering 39) 
El tiempo presente exigía cooperar con el enemigo comunista del pasado para vencer al nuevo enemigo común que representaba ahora el nazismo. Si bien había visto en el pasado crímenes, tragedias y locuras, reconoce ver ahora a un pueblo que protege su tierra y sus casas, donde las madres y las mujeres rezan y los niños juegan alegremente en los parques, y cuya alegría y dignidad contrasta con la brutalidad del ejército nazi. Estas fueron las palabras de Churchill:

I see Russian soldiers standing on the threshold of their native land guarding the fields which their fathers have tilled from time immemorial. I see them guarding their homes, where mothers and wives pray. Ah, yes, for there are times when all pray for the safety of their loved ones, for the return of the bread winner, of the champion of their protector. I see the 10,000 villages in Russia where the means of exitence is wrung so hardly form the soil, but where there are still primordial human joys, where maidens laugh and children play. I see advancing is upon all this the invidious onslaught of the Nazi war machine, with its clanging, heel-clicking, dandified Prussian officers, its crafty expert agents, fresh from cutting and cowing down of a dozen countries. I see also the dull, drilled, docile, brutish masses of the Hun soldiery coming on like a swarm of crawling locusts. I see the German bombers and fighters in the sky, still smarting from may a British whipping, and they are likely to find, they believe, an easier and safer prey.

Behind all this storm, I see that small group of venomous men who planned, organized and launched this cataract of horrors upon mankind. Then my mind goes back across the years to the day when Russian armies were our allies against the same deadly foe, when they fought with so much valor and constancy and helped to gain a victory, from a share of which, alas, they were, from no fault of ours, utterly cut out. (cit. en Levering 39-40)

Como dice Herring, con el "dramatic broadcast" de la noche del 22 de junio de 1941, Churchill dio la bienvenida a la Unión Soviética en la lucha contra el nazismo y exhortó al pueblo británico a dejar de lado las diferencias que en el 
pasado tuvo con la URSS, a la vez que dirigió su apelación final al presidente norteamericano Franklin D. Roosvelt para que abandonara su posición neutral que había adoptado al inicio del conflicto. Finalmente, Estados Unidos abandonó su neutralidad en junio de 1941 (Herring 2-3).

Sin embargo, por mucho que su suerte como pueblo dependiera de la defensa de Stalingrado, era muy difícil contrarrestar, de la noche a la mañana, el relato consolidado en el imaginario norteamericano sobre la Unión Soviética. Sobre todo, entre los sectores más conservadores de la sociedad, incluyendo a los católicos, que veían la URSS como una suerte de infierno ateo. Era necesario desplazar a los conservadores católicos de sus posiciones, y Roosevelt decidió entonces escribir al Papa Pío XII. La carta decía lo siguiente:

In so far as I am informed, churches in Russia are open. I belive there is a real possibility that Russia may as a result of the present conflict recognize freedom of religion in Russia, although, of course, without recognition of any offcial intervention on the part of any church in education or political matters within Russia. I feel that if this can be accomplished it will put the possibility of the restoration of real religious liberty in Russia on a much better footing than religious freedom is in Germany today. (cit. en Levering 51)

\section{Y seguidamente:}

I belive that the survival of Russia is less dangerous to religion, to the church as such, and to humanity in general than would be the survival of the German form of dictatorship. Furthermore, it is my belief that the leaders of all churches in the United States should recognize these facts clearly and should not close their eyes to these basic questions and by their present attitude on this question directly assist Germany in her present objectives. (51) 


\section{Conclusión}

La opinión hacia la Unión Soviética en los Estados Unidos se fue modificando en los años cruciales de la Segunda Guerra Mundial. Las dos potencias irreconciliables, con proyectos políticos y económicos diametralmente opuestos, parecían condenadas a entenderse: el ascenso del nazismo, su política de guerra expansionista y, sobre todo, la invasión a la Unión Soviética, constituían una seria amenaza para Europa y los Estados Unidos. Si la Unión Soviética caía, el Reino Unido y los Estados Unidos irían detrás. Había que cooperar con la URSS, ayudarla militarmente para contener al nazismo, no por altruismo, no por solidaridad entre los pueblos, sino por mera supervivencia. La alianza con el país comunista era imprescindible. Pero, ante los ojos de la opinión pública, un apoyo al antiguo enemigo comunista -que para algunos, sobre todo para los sectores más conservadores de la sociedad, pero también para el capital privado, constituía un peligro mayor que el nazismo- no iba a ser en absoluto bien visto, iba a ser cuestionado. Era necesario movilizar todos los recursos propagandísticos para modificar la opinión entre los sectores más conservadores. Libros, revistas, periódicos, radios, eventos, cine, declaraciones públicas, la mediación del Papa de Roma, todo ello contribuyó a transformar el estado de opinión y a construir "esa euforia rusófila" de la que nos habla Alejo Carpentier en La consagración de la primavera.

El modo en que el estado movilizó sus recursos para modificar la opinión de un pueblo bien merece una reflexión, pero también una llamada de alerta. Si bien es cierto que, como dice Small, de forma muy positiva, "the American mass media did a reasonably good job. At least, they offered views of Russia which were more balanced than those presented before the war" (477), el rápido cambio de opinión del pueblo norteamericano, que del rechazo inicial a la URSS pasó a "esa euforia rusófila", debería obligarnos a hacer una reflexión 
profunda acerca del poder que tienen los medios de comunicación en la construcción de subjetividades. Porque, como advierte Small, en otro lugar de su texto:

Such an inquiry, of course, involves the more general questions of how media shape, reinforce, and alter images of foreign countries, and whether the public can change its mind about a major issue in a short period of time. And, with relevance for the present, how quickly can the media transform the enemy of yesterday into the friend of tomorrow? (457)

La investigación sobre este fenómeno cultural que La consagración de la primavera de Alejo Carpentier recupera -o nos impulsa a recuperar, como se ha tratado de hacer con este artículo- involucra una serie de preguntas críticas que tienen que ver con el modo en que se producen los estados de opinión, el sentido común de una época o los relatos históricos. A través de la verticalidad de las creencias, ideas y valores. El rápido cambio de opinión sobre la Unión Soviética, vivido en Estados Unidos durante la Segúnda Guerra Mundial, y la manera en que el poder movilizó sus recursos para que este cambio en efecto se produjera, nos insta a pensar, siguiendo a Small, si lo que consideramos como nuestras opiniones son de verdad nuestras, si de verdad nos pertenecen o si, al contrario, son una suerte de "falsa conciencia" (Engels 523) funcional a los intereses de las clases dirigentes.

Por otro lado, la existencia de la producción cultural y el estado de opinión positiva del pueblo norteamericano hacia el pueblo ruso durante ese breve paréntesis de alianza entre Estados Unidos y la Unión Soviética en la Segunda Guerra Mundial, contiene otra lectura posible, menos vertical que aquella que se extrae de las conclusiones de Small. Esa otra lectura posible, que deja un espacio en esta historia para la contigencia, permite entender o leer este fenómeno como la expresión política y cultural de un pueblo que, de 
forma más o menos espontánea, y al margen de los prejuicios o las ideas que anteriormente tenía sobre la Unión Soviética, elabora una forma de pensar distinta, en consonancia con los nuevos tiempos. La nueva realidad histórica produce así mismo una nueva forma de pensar, de situarse en el mundo y de interpretarlo.

En cualquier caso, el redescubrimiento de este fenómeno a través de la literatura permite la articulación o la búsqueda de un nuevo lugar desde el que leer e interpretar la historia, fuera del marco interpretativo que nos dejó la Guerra Fría. Descubrir o redescubrir esa producción cultural nos impulsa a redefinir la historia, a tomar conciencia de que lo que asumiamos como histórico no era sino una forma de narratividad, un relato construido para legitimar una visión geopolítica del mundo -partido en dos- y unas políticas concretas para mantener ese escenario de conflicto que acaso beneficiaba a las fuerzas contingentes. La emergencia de este fenómeno y su lectura en el aquí y ahora permite desafiar el relato dominante y así mismo imaginar que la historia hubiera podido ser de otra manera; la recuperación y su posterior lectura de este fenómeno cultural nos permite regresar a él no como un lugar perdido para siempre sino como un lugar que, rememorándolo, es posible actualizar.

La consagración de la primavera de Alejo Carpentier demuestra que la literatura, en ocasiones, hace posible el viaje a ese pasado frustrado, a ese pasado que fue, pero cuyas condiciones de posibilidad dejaron rápidamente de serlo; a ese pasado no amortizado en el presente. La literatura, a veces, y acaso la novela de Carpentier sea un ejemplo de ello, contiene el potencial político necesario para hacer emerger una memoria que ayude a recuperar y a actualizar los acontecimientos del pasado, a cuestionar la constitución de los regímenes de verdad y a repensar la relación entre narración y poder. 


\section{Notas}

Esta investigación se inició durante una estancia de investigación en la New York University en la primavera-verano de 2016. Agradezco profundamente a la profesora Jo Labanyi que cursara la invitación. La continuación de la investigación ha sido posible gracias al programa internacional de investigación post-doctoral "Move-in Louvain" de la Université catholique de Louvain.

1 "El ocaso de Europa" es el título de un artículo publicado en 1941 por Carpentier en la revista cubana Carteles.

2 En verdad, tal trabalenguas no se pudo haber producido del modo explicado por Carpentier, ni se produciría el calambur "you are", debido a que en inglés las siglas de la Unión Soviética no son URSS, como en español, sino USSR [Union of Soviet Socialist Republics]. Aunque no es frecuente, a veces Carpentier también se equivoca.

3 De hecho, el libro se publica con esta advertencia al lector sobre la apariencia del libro:

ABOUT THE APPEARANCE OF BOOKS IN WAR TIME:

A recent ruling by the War Production Board has curtailed the use of paper by book publishers in 1943. in line with this ruling and in order to conserve materials and manpower, we are cooperating by:

1. Using lighter-weight paper which reduces the bulk of our books substantially. 2. Printing books with smaller margins and with more words to each page. Result: fewer pages per book.

Slimmer and smaller books will save paper and plate metal and labor. We are sure that readers will understand the publishers' desire to co-operate as fully as possible with the objectives of the War Production Board and our government. 


\section{Obras citadas}

Bambery, Chris. Historia marxista de la Segunda Guerra Mundial. Pasado \& Presente, 2015.

Beevor, Antony. La Segunda Guerra Mundial. Pasado \& Presente, 2012.

Benjamin, Walter. Atlas/Constelaciones. Círculo de Bellas Artes, 2010.

Bourke-White, Margaret. Shooting the Russian War. Simon and Schuster, 1942.

Carpentier, Alejo. La consagración de la primavera. 1978. Edición de David Becerra Mayor. Akal, 2015.

—. "La novela latinoamericana en vísperas de un nuevo siglo." En Roberto González Echevarría, comp. Historia y ficción en la narrativa latinoamericana. Monte Ávila, 1984, pp. 19-48.

Davies, Joseph E. Mission to Moscow. Simon and Schuster, 1941.

Dennett, Raymond, y Joseph E. Johnson, editores. Negotiating with the Russians. World Peace Foundation, 1951.

Engels, Friedrich. Carta de Friedrich Engels a Franz Mehring. Londres, 14 de julio de 1893. En Karl Marx y Friedrich Engels, Obras escogidas. Progreso, 1974, p. 523.

González Echevarría, Roberto. “Historia y alegoría en la narrativa de Carpentier." Cuadernos Americanos, no. 1, enero-febrero 1980, pp. 200-20.

Herring Jr., George C. Aid to Russia, 1941-1946: Strategy, Diplomacy, the Origins of the Cold War. Columbia University Press, 1973.

Levering, Ralph B. American Opinion and the Russian Alliance (1939-1945). The University of North Carolina Press, 1976.

Lovenstein, Meno. American Opinion of Soviet Russia. American Council on Public Affairs, 1941.

Margolin, Arnold D. From A Political Diary. Russia, the Ukraine, and America (1905-1945). Columbia University Press, 1946.

New York Times. "Best War Fact Film Opens" (31 de diciembre de 1942). ProQuest Historical Newspapers: The New York Times with Index, p. 21.

—. "Prokofieff, Soviet Composer, Sends Greetings to U.S. for Colleagues" (2 de enero de 1943). ProQuest Historical Newspapers: The New York Times with Index, p. 15.

—. "By Telephone to THE NEW YORK TIMES" (7 de enero de 1943). ProQuest Historical Newspapers: The New York Times with Index, p. 2. 
—. "Christmas Eve in Russia" (7 de enero de 1943). ProQuest Historical Newspapers: The New York Times with Index, p. 3.

—. "Concert Commitee Head" (7 de enero de 1943). ProQuest Historical Newspapers: The New York Times with Index, p. 25.

Pauwels, Jacques R. El mito de la guerra buena. Hiru, 2002.

Reston, James B. Prelude to Victory. Afred A Knopf, 1943.

Rodríguez Puértolas, Julio. Introducción. La consagración de la primavera. Castalia, 1998, pp. 9-88.

Small, Melvin. "How We Learned to Love the Russians: American Media and the Soviet Union During the World War II." The Historian, vol. 36, no. 3, mayo 1974, pp. 45578.

Walsh, Warren B. "American Attitudes toward Russia." The Antioch Review, vol. 7, no. 2, verano, 1947, pp. 183-190.

Webb, Sidney, y Beatrice Webb. The truth about Soviet Russia. Green and Co. 1942.

Willen, Paul. “Who 'Collaborated' with Russia.” The Antioch Review, vol. 14, no. 3, otoño 1954, pp. 259-83.

Williams, Albert Rhys. The Russians: The Land, The People, and Why They Fight. Harcourt, Brace and Company, 1943.

Williams, Raymond. Marxismo y literatura. Las cuarenta, 2009.

Willkie, Wendell L. One World. Simon and Schuster. 1943. 